Subscriber access provided by King Abdullah University of Science and Technology Library

\title{
Article
}

\section{Atomic-Monolayer Two-Dimensional Lateral Quasi-Heterojunction Bipolar Transistors with Resonant Tunneling Phenomenon}

Che-Yu Lin, Xiaodan Zhu, Shin-Hung Tsai, Shiao-Po Tsai, Sidong Lei, Ming-Yang Li, Yumeng Shi, LainJong Li, Shyh-Jer Huang, Wen-Fa Wu, Wen-Kuan Yeh, Yan-Kuin Su, Kang L. Wang, and Yann-Wen Lan ACS Nano, Just Accepted Manuscript • DOI: 10.1021/acsnano.7b05012 • Publication Date (Web): 04 Oct 2017

Downloaded from http://pubs.acs.org on October 10, 2017

\section{Just Accepted}

"Just Accepted" manuscripts have been peer-reviewed and accepted for publication. They are posted online prior to technical editing, formatting for publication and author proofing. The American Chemical Society provides "Just Accepted" as a free service to the research community to expedite the dissemination of scientific material as soon as possible after acceptance. "Just Accepted" manuscripts appear in full in PDF format accompanied by an HTML abstract. "Just Accepted" manuscripts have been fully peer reviewed, but should not be considered the official version of record. They are accessible to all readers and citable by the Digital Object Identifier (DOI®). "Just Accepted" is an optional service offered to authors. Therefore, the "Just Accepted" Web site may not include all articles that will be published in the journal. After a manuscript is technically edited and formatted, it will be removed from the "Just Accepted" Web site and published as an ASAP article. Note that technical editing may introduce minor changes to the manuscript text and/or graphics which could affect content, and all legal disclaimers and ethical guidelines that apply to the journal pertain. ACS cannot be held responsible for errors or consequences arising from the use of information contained in these "Just Accepted" manuscripts. 


\title{
Atomic-Monolayer Two-Dimensional Lateral Quasi- Heterojunction Bipolar Transistors with Resonant Tunneling Phenomenon
}

\author{
Che-Yu Lin ${ }^{1}$,Xiaodan Zhu ${ }^{2}$,Shin-Hung Tsai ${ }^{2}$,Shiao-Po Tsai ${ }^{2}$,Sidong Lei ${ }^{2}$,Ming-Yang Li ${ }^{3}$,Yumeng Shi ${ }^{4}$, \\ Lain-Jong Li ${ }^{5}$,Shyh-Jer Huang ${ }^{6}$,Wen-Fa Wu ${ }^{7}$,Wen-Kuan Yeh ${ }^{7}$, Yan-Kuin Su ${ }^{1,8}$,
}

Kang L.Wang ${ }^{2}$, Yann-Wen Lan ${ }^{9, *}$

${ }^{1}$ Institute of Microelectronics and Advanced Optoelectronic Technology Center, National Cheng Kung University, Tainan 701, Taiwan.

${ }^{2}$ Department of Electrical Engineering, University of California at Los Angeles, Los Angeles, California, United States.

${ }^{3}$ Research Center for Applied Sciences, Academia Sinica, Taipei, Taiwan.

${ }^{4}$ SZU-NUS Collaborative Innovation Center for Optoelectronic Science and Technology, Shenzhen University, Shenzhen 518060, China

${ }^{5}$ Physical Sciences and Engineering Division, King Abdullah University of Science and Technology (KAUST), Thuwal, Kingdom of Saudi Arabia.

${ }^{6}$ Advanced Optoelectronic Technology Center, National Cheng Kung University, Tainan 701, Taiwan.

${ }^{7}$ National Nano Device Laboratories, National Applied Research Laboratories, Hsinchu 30078, Taiwan.

${ }^{8}$ Department of Electrical Engineering, Kun Shan University, Tainan 710, Taiwan.

${ }^{9}$ Department of Physics, National Taiwan Normal University, Taipei 11677, Taiwan

Tel: +886-2-77346094, Fax: +886-2-29326408, Email: ywlanblue@gmail.com; ywlan@ntnu.edu.tw 
Abstract-High-frequency operation with ultra-thin, lightweight and extremely flexible semiconducting electronics are highly desirable for the development of mobile devices, wearable electronic systems and defense technologies. In this work, the experimental observation of quasi-heterojunction bipolar transistors utilizing a monolayer of the lateral $\mathrm{WSe}_{2}-\mathrm{MoS}_{2}$ junctions as the conducting p-n channel is demonstrated. Both lateral n-p$n$ and $p-n-p$ heterojunction bipolar transistors are fabricated to exhibit the output characteristics and current gain. A maximum common-emitter current gain of around 3 is obtained in our prototype two-dimensional quasi-heterojunction bipolar transistors. Interestingly, we also observe the negative differential resistance in the electrical characteristics. A potential mechanism is that the negative differential resistance is induced by resonant tunneling phenomenon due to the formation of quantum well under applying high bias voltages. Our results open the door to two-dimensional materials for high-frequency, high-speed, high-density and flexible electronics.

Keywords: 2D materials, heterojunction bipolar transistors, resonant tunneling phenomenon, lateral junction, atomic layered, 
One of the major transistors is bipolar junction transistor (BJT) that is formed by connecting two opposite junction diodes and utilizes both electron and hole charge carriers. It is a three-terminal device represented separately by emitter - base and collector, and is a critical component in many analog, digital, and sensor applications. BJTs are manufactured in two types, NPN and PNP, and the two complementary configuration transistors could be fabricated in the same circuit which could make the circuit design more flexible. The amplification of current is the basic function of a BJT. ${ }^{1}$ This allows BJTs to be used as amplifiers or switches, giving them wide applicability in consumer electronics, examples of applications including communication products, computers, audio-visual and sound equipment, and various instruments. The heterojunction bipolar transistor (HBT) is very similar to the BJT, but uses different semiconductor materials with different bandgaps for the emitter and base regions instead. ${ }^{2,3}$ Compared to BJT, HBT can be operated at very high frequencies, up to several hundred GHz. It is commonly used in radio-frequency (RF) systems such as RF power amplifiers in cellular phones. ${ }^{4,5}$ Traditional materials used for epitaxial layers of HBT include silicon/silicon-germanium alloys, ${ }^{6}$ aluminum gallium arsenide/gallium arsenide, and indium phosphide/indium gallium arsenide.

The advent of atomically thin two-dimensional (2D) crystals has sparked a paradigm shift in nanotechnology. ${ }^{7,8}$ Since the last decade, we are capable of truly exploring and implementing device concepts at the ultimate physical thickness limit in addition to having at our disposal a myriad of 2D materials, each of which exhibits distinctive electronic and optical properties. ${ }^{9}$ There exist many $2 \mathrm{D}$ materials with energy bandgaps in the range between 1 and $2 \mathrm{eV}$. By carefully mixing and matching materials with different bandgap values, it can provide the potential barriers needed to limit the injection of holes from the base into the emitter region and thus enhance the operation frequency. Further, 2D materials are good candidates to replace traditional semiconductors for resonant tunneling diodes (RTDs) since that realizing the negative differential resistance (NDR) phenomenon in a resonant tunneling diode (RTD) at room temperature has been challenging due to carrier scattering related to interfacial imperfections. ${ }^{10-12}$ This is unavoidable in the study of conductive semiconductor heterostructures using advanced epitaxial growth techniques. However, one of 2D material 
features ${ }^{13-15}$ is no out-of-plane dangling bonds and pristine interfaces. It shows an appealing alternative to traditional semiconductors, and could effectively eliminate the interfacial imperfections which could break through the limit room of low temperature NDR performance. One of the $2 \mathrm{D}$ semiconductor materials is transition metal dichalcogenides (TMDs) which is very attractive because they can be atomically thin, ${ }^{16}$ possess high conductivity, high thermal conductivity, high mechanical strength, transparency and so on. Several devices have already been demonstrated ${ }^{17-19}$ due to their exceptional electronic and optical properties. If these TMD materials are in the monolayer form, they would be direct bandgap which are beneficial for optoelectronic devices. ${ }^{20-23}$ When the $\mathrm{MoS}_{2}$ and $\mathrm{WSe}_{2}$ materials are stacked together, it would exhibit type II band alignment between the two kinds of materials, ${ }^{24-26}$ where electrons in the $\mathrm{WSe}_{2}$ conduction band transfer to the conduction band of $\mathrm{MoS}_{2}$ and the excited holes in $\mathrm{MoS}_{2}$ valence band transfer to the valence band of WSe $\mathrm{W}_{2}$ It indicates the $\mathrm{MoS}_{2}$ exhibits an n-type behavior while the $\mathrm{WSe}_{2}$ layer shows a p-type behavior. Recently, we have demonstrated a vertical hot electron transistor based on TMD semiconductor $\operatorname{MoS}_{2}{ }^{13}$ which is a pioneer work of $2 \mathrm{D}$ electronics and its function is similar to a bipolar junction transistor. Lately, the lateral heterojunction $\left(\mathrm{WSe}_{2}-\mathrm{MoS}_{2}\right.$ ) with an atomically sharp interface has been successfully grown and reported. ${ }^{27}$ It is an ideal platform to study 2D-HBT in the advanced stage. In this work, we demonstrate a quasi-heterojunction bipolar transistor constructed solely of a monolayer of lateral $\mathrm{WSe}_{2}-\mathrm{MoS}_{2}$ heterojunctions. To the best of our knowledge, this is the creative demonstration of utilizing a monolayer lateral heterostructure material as the channel for the study of HBT in an n-p-n and p-n-p configuration. Furthermore, NDR behavior is observed, which is induced by resonant tunneling phenomenon due to the formation of quantum well under applying high bias voltages.

\section{RESULTS AND DISCUSSION}

For the synthesis of $\mathrm{WSe}_{2}-\mathrm{MoS}_{2}$ lateral heterostructure, a monolayer $\mathrm{MoS}_{2}$ was grown using chemical vapor deposition (CVD) on top of sapphire substrates. The $\mathrm{MoS}_{2}$ monolayer flakes are then etched into stripes of a few micrometers in width using $\mathrm{O}_{2}$ plasma. Subsequent $\mathrm{WSe}_{2}$ growth using CVD results in preferred growth in 
the spaces between the $\mathrm{MoS}_{2}$ ribbons to form triangular flakes. The detailed growth process of $\mathrm{WSe}_{2}-\mathrm{MoS}_{2}$ lateral heterostructure is reported in the previous work. ${ }^{27} \mathrm{O}_{2}$ plasma is again used to define ribbons with alternating materials for ease of device fabrication. Fig. 1 shows the characterization of the lateral $\mathrm{WSe}_{2}-\mathrm{MoS}_{2}$ heterostructure in one of the ribbons. The atomic force microscope (AFM) image and the corresponding optical micrograph of the ribbon are locally shown in Fig. 1(a). While the AFM image clearly indicates a thickness of a monolayer lateral heterostructure to be about $0.8 \mathrm{~nm}$ (Fig. 1(b)), the optical micrograph faintly displays two colors in the ribbon, revealing the lateral 2D ribbon with two kinds of materials. Due to the different adhesion between AFM tip and 2D materials, the phase image of AFM can exhibit a clear contrast between WSe ${ }_{2}$ and $\mathrm{MoS}_{2}$ as shown in Fig. 1(c). The height profile of the lateral junction between two materials marked in (c), showing an ultra smooth interface in Fig. 1(d). Further, Raman spectrums for the $\mathrm{MoS}_{2}$ and $\mathrm{WSe}_{2}$ are also presented to confirm the monolayer material in Fig. 1(e) and Fig. 1(f), respectively. Fig. 2(a), 2(b) and 2(c) are the process flow of the device fabrication. Fig. 2(a) shows the top view in one of the patterned ribbons where two distinct colors are present to reveal $\mathrm{MoS}_{2}$ (n-type) and $\mathrm{WSe}_{2}$ (p-type). Other patterned ribbons are shown in SF1 in supporting information. We deposit the contact metals $\mathrm{Ti} / \mathrm{Au}$ on the top of the $\mathrm{MoS}_{2}$ material as shown in Fig. 2(b) and then the contacts metals $\mathrm{Pd} / \mathrm{Au}$ are placed on top of $\mathrm{WSe}_{2}$ layer. Metal contacts are defined using e-beam lithography and standard lift-off processes. Ti/Au is used for contacts to $\mathrm{MoS}_{2}$ regions while $\mathrm{Pd} / \mathrm{Au}$ is used for $\mathrm{WSe}_{2}$ regions to ensure good contacts. Fig. 2(c) exhibits the optical micrograph of a complete lateral heterojunction bipolar transistor in one of our devices, showing an n-p-n-p lateral heterojunction. Fig. 2(d) shows a schematic plot of a heterojunction bipolar transistor in which yellow ribbons are the $\mathrm{MoS}_{2}$ monolayers while red ribbon is the $\mathrm{WSe}_{2}$ monolayer. Electrical characterization in this work is carried out at room temperature using a semiconductor parameter analyzer (Keithley 4200). In this particular study, a common-emitter configuration was employed during the electrical measurement.

The p-n junction consists of two semiconductor regions with opposite doping type, which is the basic property in both BJT and HBT. Regarding to the lateral p-n junction in 2D materials, an atomically sharp 
interface between two materials can be seen in reference ${ }^{27}$ and the fundamental electrical characterization for the junctions is presented below. Fig. 3(a) shows the optical micrograph of one of our lateral (n-p-n) quasiheterojunction bipolar transistors. Fig. 3(b) shows the current-voltage characteristics for the n-p junction in which the curve in blue color is semi-logarithmic scale while the curve in black color shows the characteristic in the linear plot. It exhibits that current is low in the forward bias whereas current is abruptly increase in the reverse bias. This rectifying characteristic is displayed to support lateral n-p junction formation between $\mathrm{MoS}_{2}$ and $\mathrm{WSe}_{2}$. Since p-n junction is very sensitive to the light, the photo-response characteristic is shown in Fig. 3(c) for the p-n junction in dark and under illumination at different intensities. It indicates both the open-circuit voltage and short-circuit current increase with increasing light intensity, operating as a photovoltaic device in the p-n junction. Electrical characteristics in both Fig. 3(b) and 3(c) independently confirm the formation of the lateral p-n junction in our samples. The electrical bandgap of the $\mathrm{MoS}_{2}$ and $\mathrm{WSe}_{2}$ monolayers are about $2.34 \mathrm{eV}$ and $2.46 \mathrm{eV}$, respectively. ${ }^{28}$ The corresponding energy band diagram of the lateral heterojunction bipolar transistors is shown in Fig. 3(d). The energy between the bottom edge of the conduction band and the Fermi level in $\mathrm{MoS}_{2}$ is about $0.216 \mathrm{eV}$ while the energy between the edge of the valence band and Fermi level in $\mathrm{WSe}_{2}$ is about $0.035 \mathrm{eV}$. Due to the differences in electron affinity and bandgap between monolayer $\mathrm{MoS}_{2}$ and $\mathrm{WSe}_{2}$, abrupt heterojunctions might also exist in our lateral heterojunction bipolar transistors. It may form the resonant tunneling of the two-dimensional electron gas or two-dimensional hole gas. By choosing different 2D materials, it is possible to have a larger band step resulting in a larger hole or electron barrier for the enhancement of the device performance.

Another device is shown in Fig. 4(a) and Fig. 6(a) to demonstrate the lateral n-p-n and p-n-p quasiheterojunction bipolar transistors in a single ribbon flake, respectively. Fig. 4(b) shows the Gummel plot in the n-p-n quasi-heterojunction bipolar transistor which was used as a figure of merit when analyzing bipolar transistor. The collector and base currents are exhibited in log-scale as a function of $\mathrm{V}_{\mathrm{BE}}$ at a fixed output voltage of $\mathrm{V}_{\mathrm{CE}}=10 \mathrm{~V}$. This plot confirms that the input base current is directly amplified to the output collector 
current. Fig. 4(c) shows the common-emitter output characteristics of the lateral n-p-n quasi-heterojunction bipolar transistor. The collector current is shown as a function of $\mathrm{V}_{\mathrm{CE}}$ at different $\mathrm{V}_{\mathrm{BE}}$ bias. When the lateral np-n quasi-heterojunction bipolar transistor is operating in the small bias, it would be in the saturation mode. In this mode, the base-emitter junction and base-collector junction are both in the forward bias and the current of the collector would not be controlled by the bias of the base-emitter junction. Moreover, when the $\mathrm{V}_{\mathrm{CE}}$ is large enough, it would be in the forward active region in which the base-collector junction would transform to reverse bias and the current of the collector would keep almost constant. These two operating regions are shown in Fig. 4(c) and similar behavior in another device can be seen in SF1 in the supporting information. Furthermore, once the $\mathrm{V}_{\mathrm{BE}}$ is increased, the collector current is increased due to lowering of the barrier between emitter and base. The common-emitter current gain $\left(\beta=\mathrm{I}_{\mathrm{C}} / \mathrm{I}_{\mathrm{B}}\right)$ can be determined by taking the ratio of the collector current to the base current at a fixed $V_{\mathrm{BE}}$. Fig. 4(d) shows the common-emitter current gain $(\beta)$ as a function of $\mathrm{V}_{\mathrm{BE}}$ at $\mathrm{V}_{\mathrm{CE}}=10 \mathrm{~V}$. A maximum common-emitter current gain of around 3 with collect current density of $0.0126 \mathrm{~A} / \mathrm{cm}^{2}$ in our devices is achieved and it is not much dependent of $\mathrm{V}_{\mathrm{BE}}$. We speculate that this is because the process is mainly dominated by the application of large collector voltages. The barrier for electrons in this condition is effectively low between base and emitter regions so that current gain is not much affected by the base voltage increased. It is noted that the collect current $\left(\mathrm{I}_{\mathrm{C}}\right)$ slightly fluctuates at small $\mathrm{V}_{\mathrm{BE}}(\mathrm{Fig}$. $4(\mathrm{c}))$. We attribute this to the interfacial band bending of the heterojunction in the resonant tunneling of the twodimensional electron gas or two-dimensional hole gas. Under this hypothesis, the electrons (holes) are free to move in two dimensions ( $\mathrm{x}$ - and $\mathrm{y}$-direction), but confined in the third (z-direction). It will lead to the energies separated and thus faintly affect the electrical transports behavior. Interestingly, when the base bias is much increased, the output characteristics of the lateral n-p-n quasi-heterojunction bipolar transistor present a negative differential resistance (NDR) phenomenon at positive bias region. We think this NDR behavior is dominated to resonant tunneling mechanism and a related schematic plot with fundamental principle and theoretical derivation is presented in SF2 in supporting information. When the size of the base channel width is 
less than or equal to the electrons of the deBroglie wavelength $\lambda_{\mathrm{d}}$, the channel will be confined to form discrete energy states, which is called quantum confined effect. The wavelength of an electron $\left(\lambda_{d}\right)$ is related to its energy $(E)$ described by ${ }^{29}$

$\lambda_{d}=\frac{h}{p}=\frac{h}{\sqrt{2 m^{*} E}} \approx \frac{h}{\sqrt{2 m^{*} k_{B} T}}$

where $h$ is the Planck constant, $p$ is the momentum, $m^{*}$ is the effective-mass of an electron in a semiconductor, $k_{B}$ is the Boltzmann constant, $T$ is the test temperature. In our n-p-n quasi-heterojunction bipolar transistors case, the estimated deBroglie wavelength of the $\mathrm{WSe}_{2}$ is around $13 \mathrm{~nm}$ with an effective mass $\left(m^{*}\right)$ of $0.33 m_{0}{ }^{30}$ at room temperature. It means that when the region of the $\mathrm{WSe}_{2}$ layer is close or less to deBroglie wavelength, the quantum well would be formed. This situation may be happened at relatively large $V_{B E}$ and $V_{C E}$ applied in our devices. The increasing $\mathrm{V}_{\mathrm{BE}}$ would decrease the base energy band so that the base region would form the well ${ }^{31}$ which is relative to the emitter and collector. The widths of depletion layer between base and collector regions are gradually increased to narrow the base region as we steadily increase bias voltage in $\mathrm{V}_{\mathrm{CE}}$, leading to the quantum confinement in the base region of $\mathrm{WSe}_{2}$ layer. To estimate the depletion width between base and collector regions, the width of the quasi-heterojunction depletion layer $W_{D}$ is defined below: ${ }^{32}$

$$
W_{D}=\sqrt{\frac{2 N_{D} \varepsilon_{1} \varepsilon_{2}\left(V_{b i}-V_{F}\right)}{q N_{A}\left(\varepsilon_{1} N_{D}+\varepsilon_{2} N_{A}\right)}}
$$

where the $N_{D}$ is the donor impurity concentration of $\mathrm{MoS}_{2}$, the $N_{A}$ is the acceptor impurity concentration of $\mathrm{WSe}_{2}, \varepsilon_{1}$ is the dielectric constant of the narrow bandgap material $\mathrm{MoS}_{2}, \varepsilon_{2}$ is the dielectric constant of the wide bandgap material $\mathrm{WSe}_{2}, V_{b i}$ is built-in potential, $V_{F}$ is the forward voltage of the base and collector junction. In our case, the width of the $\mathrm{WSe}_{2}$ is about $1.3 \mu \mathrm{m}$ determined from device image (see Fig. 2(a)) and the depletion layer between base and emitter regions can be neglected because of the large forward bias applied. Assuming that $N_{D}$ is $2 \times 10^{9} \mathrm{~cm}^{-2}$ (this is close to the value in reference ${ }^{33}$ ) at $0.8 \mathrm{~nm}$ (film thickness in Fig. $1(\mathrm{~b})$ ) and $V_{b i}$ is about $0.8 \mathrm{~V},{ }^{34} V_{F}=-4.47 \mathrm{~V}, N_{A}$ is $2.2 \times 10^{12} \mathrm{~cm}^{-2},{ }^{35} \varepsilon_{1}$ is $7 \varepsilon_{0},{ }^{36} \varepsilon_{2}$ is $4.5 \varepsilon_{0},{ }^{37}$ the estimated depletion layer is 
around $1.2964 \mu \mathrm{m}$ which is comparable with the base width of the $\mathrm{WSe}_{2}$. Consequently, the base width is potentially lesser than deBroglie wavelength and form the quantum states.

From our observations shown in Fig 5(a), the larger base and collector bias applied indeed result in the quantum resonant tunneling behavior. This is because the larger base bias would let the well deeper while the larger collector bias would provide the depletion region larger, as shown in Fig. 5(b), so that the base width will be comparable or less than de Broglie wavelength. Under this condition, it is capable to form the discrete energy and generate the quantum confinement effect in room temperature. The lateral $n-p$ and $p-n$ junction between $\mathrm{MoS}_{2}$ and $\mathrm{WSe}_{2}$ in n-p-n quasi-heterojunction bipolar transistors act as both tunnel barriers. Once the energy in the emitter region is higher than the energy in the quantum well (base region), we can observe the resonant tunneling behavior. Fig. 5(c) shows the confined state in the middle region $\left(\mathrm{WSe}_{2}\right)$ is getting close to align with the source Fermi level in the left region $\left(\mathrm{MoS}_{2}\right)$ when collector current approach to current peak maximum at certain large amount of $\mathrm{V}_{\mathrm{CE}}$ applied. When the $\mathrm{V}_{\mathrm{CE}}$ is further increased, this confined state is moving away from the source Fermi level, exhibiting current decreased as seen in Fig. 5(d). With further increase of the $V_{C E}$, the collector current will increase because the next confined state is moving close to the source Fermi level, displaying current increased again as shown in Fig. 5(e). This behavior is repeatable and reproducible. Another device that exhibits the similar NDR behavior is shown in SF3 in supporting information. The detailed estimation of energy states and Fermi level tuning for the resonant tunneling phenomenon in a n-p-n lateral quasi-heterojunction bipolar transistor is also presented in SF4 in supporting information. With comparison between estimation of energy states and experimental observation, some of resonant peaks are not evident or missing, it is due to the fact that thermal broadening and fluctuations exist in room temperature. In addition, we also note that when the $\mathrm{V}_{\mathrm{BE}}$ is increased at relatively large bias, the current of the collector would not increase like common bipolar junction transistors. We think that it might be due to the more base bias the deeper well which lead to the smaller resonant energies ${ }^{38}$. 
Besides, the lateral p-n-p quasi-heterojunction bipolar transistor is also present in the same flake of the ribbon as shown in Fig. 6(a). The similar results for Gummel plot, output characteristics and common-emitter current gain are displayed in Fig. 6(b), 6(c) and 6(d), respectively. We note that the output characteristic is not saturated even if the collector voltages are applied to $10 \mathrm{~V}$. Furthermore, the common-emitter current gain $(\beta)$ is about 2 with collect current density of $0.004 \mathrm{~A} / \mathrm{cm}^{2}$ and it is slightly dependent on base voltage applied. We attribute this discrepancy between n-p-n and p-n-p transistor to the fact that the barrier for holes transport in the p-n-p case is steeper than the barrier for electrons in the n-p-n case, as seen in Fig. 3(d). Additionally, we speculate that current gain can be greatly improved by reducing or doping the base region since it is about 1.3 $\mu \mathrm{m}$ in the base width of our devices, which is too long to be effectively traversed by minority carriers ${ }^{39}$. Lastly our device performance and properties (the measured current gain, collect current density and thickness) is compared with that of Si based transistors and other materials, which is summarized in Table 1. The advantages in our transistors are the demonstration of ultra-thin transistor for the way to scale devices down in the future. Further, the concept of these lateral transistors can guide into the development of thin film silicon-on-insulator technology for device application. However, the disadvantages in our current devices are low current gain and low current density, which are needed to improve for practical application. If the base width is narrower than the diffusion length (which has been estimated to be about $1 \mu \mathrm{m}{ }^{27}$ ) and the doping in the emitter region could be increased for more carriers to traverse, we believe high current gain 2D lateral heterojunction bipolar transistor could be realized in the future.

\section{CONCLUSION}

We have demonstrated quasi-heterojunction bipolar transistors using a monolayer of the lateral heterojunction transition metal dichalcogenide materials, namely $\mathrm{WSe}_{2}-\mathrm{MoS}_{2}$. Two types of the lateral quasiheterojunction bipolar transistor, n-p-n and p-n-p, are meticulously fabricated and studied in the same piece of a flake in order to compare the device performance between them. From the result of electrical characteristics, the maximum common-emitter current gain achieved is around 3 in our prototype two-dimensional quasi- 
heterojunction bipolar transistors. Furthermore, we also observe that the negative differential resistance (NDR) in our devices. The NDR behavior is caused by resonant tunneling phenomenon due to the formation of quantum well under applying high bias voltages in the base region and collector region. The results are fascinating and promising for the future development of electronics based on the concept of quasiheterojunction bipolar transistor using a monolayer 2D lateral heterojunctions.

\section{METHODS /EXPERIMENTAL}

$\mathrm{MoS}_{2}-\mathrm{WSe}_{2}$ synthesis. The 2D lateral $\mathrm{WSe}_{2}-\mathrm{MoS}_{2}$ heterojunction was synthesized on c-plane sapphire substrates by chemical vapor deposition (CVD).We synthesized this type of the device by two-step growth. Firstly, the $\mathrm{WSe}_{2}$ growth proceeds from $\mathrm{WSe}_{2}$ seeds, followed by van der Waals epitaxy at the higher growth temperature $\left(925^{\circ} \mathrm{C}\right)$. Secondly, to avoid the alloy reaction, we performed the $\mathrm{MoS}_{2}$ monolayer growth at $755^{\circ} \mathrm{C}$ in a separate furnace by $\mathrm{MoO}_{3}$ and $\mathrm{S}$ precursors.

Raman spectra measurement. Raman spectrums were taken by a Horiba HR800 system with laser excitation wavelength of $532 \mathrm{~nm}$.

AFM and electrical characterization of devices. All AFM-based methods employed the Dimension Icon scanning probe microscope (Bruker Nano, Inc.) in ambient conditions using SiN probes (ScanAsyst-Air, calibrated spring constants of $0.3-0.5 \mathrm{~N} \mathrm{~m}^{-1}$ and nominal tip radius of $2 \sim 5 \mathrm{~nm}$ ). Cantilever spring constants were calibrated using the Sader method. Topographic characterization was carried out in the PeakForce Tapping mode. Electrical properties of fabricated devices were measured with a semiconductor parameter analyser (Keithley 4200) at room temperature.

\section{ACKNOWLEDGMENT}

This work was supported by the National Science Council, Taiwan under contract No. MOST 105-2112-M-492003-MY3. This work was also in part supported by the National Nano Device Laboratories and Core facilities at 
UCLA. We would like to acknowledge the collaboration of this research with King Abdul-Aziz City for Science and Technologies (CEGN).

\section{AUTHOR CONTRIBUTIONS}

YannWen Lan, Xiaodan Zhu, Shin-Hung Tsai, Shiao-Po Tsai and Sidong Lei fabricated the devices and carried out OM, AFM, Raman measurements and the electrical characterization. Ming-Yang Li, Yumeng Shi and LainJong Li synthesized the 2D heterostructures. Yann-Wen Lan ,Wen-Fa Wu, Wen-Kuan Yeh, Kang L.Wang, Shyh-Jer Huang and Yan-Kuin Su supervised the study. Che-Yu Lin and Yann-Wen Lan analysed the data and wrote the paper with significant inputs from all authors. All authors have read and approved the manuscript. All authors discussed the results and commented on the manuscript.

\section{COMPETING INTERESTS}

The authors declare no competing financial interests.

\section{References}

1. Sze, S. M. Physics of Semiconductor Devices, 3rd ed. Wiley-Interscience, 2007.

2. Kroemer, H.. Theory of a Wide-Gap Emitter for Transistors. Proc. IRE 1957, 45, 1535.

3. Kroemer ,H.. Heterosturcture Bipolar Transistors and Integrated Circuits. Proc. IEEE 1982, 70, 13.

4. Ali, F; Gupta, A; Higgins, A. Advances in GaAs HBT Power Amplifiers for Cellular Phones and Military Applications. IEEE MTT-S Microwave Symp Dig 1996, 61.

5. van Bezooijen, A.; Prikhodko, D.; van Roermund, A. H. M.. Biasing Circuits for Voltage Controlled GSM Power Amplifiers. Proc 11th GAAS Symp 2003, Munich.

6. Cressler, J.D.. SiGe HBT Technology: A New Contender for Si-Based RF and Microwave Circuit Applications. IEEE transactions on microwave theory and techniques 1998, Vol 46, No5, 572-589.

7. Radisavljevic, B.; Radenovic, A.; Brivio, J.; Giacometti, V.; Kis, A.. Single-Layer $\mathrm{MoS}_{2}$ Transistors. Nat. Nanotechnol. 2011, 6, 147-150.

8. Fiori, G.; Bonaccorso, F.; Lannaccone, G.; Palacios, T.; Neumaier, D.; Seabaugh, A.; Banerjee, S. K.; Colombo, L.. Electronics Based on Two-Dimensional Materials. Nature Nanotechnol. 2014 ,9, 768-779.

9. Wang, Q. H.; Kalantar-Zadeh, K.; Kis ,A.; Coleman, J. N.; Strano, M. S.. Electronic and Optoelectronics of Two-Dimensional Transition Metal Dichalcogenides. Nature Nanotechnol. 2012, 7, 699-712.

10. Shewchuk, T. J.; Chapin, P. C.; Coleman, P. D.. Resonant Tunneling Oscillations in a GaAs-Al $\mathrm{Aa}_{1-\mathrm{x}} \mathrm{As}$ Heterostructure at Room Temperature. Appl.Phys. Lett. 1985, 46, 508.

11. Tsuchiya, M.; Sakaki, H.; Yoshino, J.. Room Temperature Observation of Differential Negative Resistance in an AlAs/GaAs/AlAs Resonant Tunneling Diode. Jpn. J. Appl. Phys. 1985, 24, L466.

12. Sekiguchia, R.; Koyama, Y. ; Ouchi, T. . Subterahertz Oscillations from Triple-Barrier Resonant Tunneling Diodes with Integrated Patch Antennas. Appl. Phys. Lett. 2010 ,96, 062115. 
13. Torres Jr.,C. M.; Lan ,Y.-W.; Zeng, C.; Chen, J.-H.; Kou, X.; Navabi ,A.; Tang, J.; Montazeri, M.; Adleman ,J. R.; Lerner, M. B.; Zhong, Y.-L.; Li ,L.-J.; Chen, C.-D.; Wang, K.-L.. High-Current Gain TwoDimensional $\mathrm{MoS}_{2}$-Base Hot-Electron Transistors. Nano Letters 2015, 15, 7905-7912.

14. Novoselov, K. S.; Geim, A. K.; Morozov, S. V.; Jiang, D.; Zhang, Y.; Dubonos, S. V.; Grigorieva, I. V.; Firsov ,A. A.. Electric Field Effect in Atomically Thin Carbon Films. Science 2004, 306, 666-669.

15. Politano, A. ; Vitiello, M.S. ; Viti ,L. ; Boukhvalov, D.W. ; Chiarello ,G. . The Role of Surface Chemical Reactivity in the Stability of Electronic Nanodevices Based on Two-Dimensional Materials "Beyond Graphene" and Topological Insulators. FlatChem 2017, 60-64.

16. Novoselov ,K. S.; Jiang, D.; Schedin, F.; Booth ,T. J.; Khotkevich, V. V.; Morozov, S. V.; Geim ,A. K.. Two-Dimensional Atomic Crystals. Proc. Natl Acad. Sci. 2005,USA 102, 10451-10453.

17. Mak, K.F.; Lee, C.; Hone, J.; Shan, J.; Heinz, T.F.. Atomically Thin $\mathrm{MoS}_{2}$ : a New Direct-Gap Semiconductor. Phys. Rev. Lett. 2010, 105, 136805.

18. Lee, C.-H.; Lee, G.-H.; van der Zande, A.M.; Chen, W.; Li, Y.; Han, M.; Cui, X.; Arefe, G.; Nuckolls ,C.; Heinz, T.F.; Guo, J.; Hone, J.; Kim, P.. Atomically Thin p-n Junctions with van der Waals Heterointerfaces. Nat. Nanotechnol. 2014, 9, 676-681.

19. Britnell ,L.; Gorbachev ,R. V.; Jalil ,R.; Belle, B. D. ; Schedin, F.; Katsnelson, M. I.; Eaves, L.; Morozov, S. V.; Peres, N. M. R.; Leist, J.; Geim, A. K.; Novoselov, K. S.; Ponomarenko, L. A.. Field-Effect Tunneling Transistor Based on Vertical Graphene Heterostructures. Science 2012, 335, 947 - 950.

20. Sarkar, D.; Xie, X.; Liu, W.; Cao, W.; Kang, J.; Gong, Y.; Kraemer,S.; Ajayan, P. M.; Banerjee, K. A Subthermionic Tunnel Field-Effect Transistor with an Atomically Thin Channel. Nature 2015, 526, 91 - 95.

21. Baugher, B. W. H.; Churchill, H. O. H.; Yang, Y.; Jarillo-Herrero,P. Optoelectronic Devices Based on Electrically Tunable p-n Diodes in a Monolayer Dichalcogenide. Nat. Nanotechnol. 2014, 9, 262 - 267.

22. Pospischil, A.; Furchi, M. M.; Mueller, T. Solar-Energy Conversion and Light Emission in an Atomic Monolayer p-n Diode. Nat. Nanotechnol. 2014, 9, $257-261$.

23. Ross, J. S.; Klement, P.; Jones, A. M.; Ghimire, N. J.; Yan, J.;Mandrus, D. G.; Taniguchi, T.; Watanabe, K.; Kitamura, K.; Yao, W.; Cobden, D. H.; Xu, X. Electrically Tunable Excitonic Light-Emitting Diodes Based on Monolayer WSe 2 p-n Junctions. Nat. Nanotechnol. 2014, 9, 268 - 272.

24. Terrones, H.; Lo'pez-Uri'as, F.; Terrones, M. Novel Hetero-Layered Materials with Tunable Direct Band Gaps by Sandwiching Different Metal Disulfides and Diselenides. Sci. Rep. 2013,3, 1549.

25. Fang, H; Battaglia, C; Carraro, C; Nemsak, S; Ozdol, B; Kang, J.S.; Bechtel, H.A.; Desai ,S.B.; Kronast, F; Unal, A.A.; Conti, G; Conlon, C; Palsson, G.K.; Martin, M.C.; Minor, A.M.; Fadley, C.S.; Yablonovitch, E; Maboudian, R; Javey, A. Strong Interlayer Coupling in van der Waals Heterostructures Built from Single-Layer Chalcogenides. Proc. Natl Acad. Sci. 2014, USA 111, 6198-6202.

26. Rivera, P; Schaibley, J.R.; Jones, A.M. ; Ross, J.S.; Wu, S; Aivazian, G; Klement, P; Seyler ,K; Clark, G; Ghimire, N.J. ; Yan, J; Mandrus, D.G.; Yao, W; Xu, X. Observation of Long-Lived Interlayer Excitons in Monolayer $\mathrm{MoSe}_{2}-\mathrm{WSe}_{2}$ Heterostructures. Nat. Commun. 2015,6, 6242.

27. Li, M.-Y.; Shi, Y; Cheng, C.-C.; Lu, L.-S.; Lin, Y.-C.; Tang, H.-L.; Tsai, M.-L.; Chu, C.-W.; Wei, K.H.; He, J.-H.; Chang, W.-H.; Suenaga, K; Li, L.-J. .Epitaxial Growth of a Monolayer WSe -MoS $_{2}$ Lateral p$\mathrm{n}$ Junction with an Atomically Sharp Interface. Science 2015, 349(6247), 524-528.

28. Chiu, M.-H.; Li, M.-Y.; Zhang, W; Hsu, W.-T.; Chang, W.-H.; Terrones, M; Terrones, H; Li, L.J.. Spectroscopic Signatures for Interlayer Coupling in $\mathrm{MoS}_{2}-\mathrm{WSe}_{2}$ van der Waals Stacking. ACS NANO 2014,VOL. 8, NO. 9, 9649-9656.

29. Griffiths, David J., Introduction to Quantum Mechanics, 2nd Edition. Pearson Education, 2005, Problem 1.18 .

30. Liu, W.; Cao, W.; Kang, J.; Banerjee, K.. High-Performance Field-Effect-Transistors on Monolayer WSe 2. ECS Transactions 2013, 58 (7) 281-285.

31. Chou, S.Y. ; Allee, D.R. ; Pease, R.F. ; Harris, J.S. . Lateral Resonant Tunneling Transistors Employing Field-Induced Quantum Wells and Barriers. IEEE 1991, Vol. 79, NO. 8. 
32. Lundstrom, M., Note on Heterostructure fundamentals, 1995,P.14.

33.Carvalho, A.; Castro Neto, A. H.. Donor and Acceptor Levels in Semiconducting Transition Metal Dichalcogenides. ,Phys. Rev. B 2013. 89, 081406(R).

34. Britnell, L.; Ribeiro, R. M.; Eckmann, A.; Jalil, R.;Belle, B. D.; Mishchenko, A.; Kim, Y.-J.; Gorbachev, R. V.; Georgiou, T.; Morozov, S. V. ;Grigorenko ,N.; Geim, A. K.; Casiraghi, C.; Castro Neto, A. H.; Novoselov ,K. S.. Strong Light-Matter Interactions in Heterostructures of Atomically Thin Films. Science 2013, VOL 340, 14.

35. Kang, D.H.; Shim, J; Jang, S.K.; Jeon, J; Jeon, M.H.; Yeom, G.Y.; Jung, W.S.; Jang, Y.H.; Lee, S; Park, J.H.. Controllable Nondegenerate $\mathrm{p}$ ? Type Doping of Tungsten Diselenide by Octadecyltrichlorosilane. ACS NANO 2015, VOL. 9 , NO. 2 , 1099-1107.

36. Salmani-Jelodar, M.; Tan, Y; Klimeck, G . Single Layer $\mathrm{MoS}_{2}$ Band Structure and Transport. ISDRS 2011 - http://www.ece.umd.edu/ISDRS2011.

37.Kumar, A.; Ahluwalia, P. K. Tunable Dielectric Response of Transition Metal Dichalcogenides $\mathrm{MX}_{2}(\mathrm{M}=\mathrm{Mo}$, W; X=S, Se, Te): Effect of Quantum Confinement. Physica B 2012,407, 4627-4634.

38. Nguyen ,H. S.; Vishnevsky ,D.; Sturm, C.; Tanese, D.; Solnyshkov, D.; Galopin, E.; Lema1 tre ,A.; Sagnes ,I.; Amo, A.; Malpuech, G.; Bloch, J.. Realization of a Double-Barrier Resonant Tunneling Diode for Cavity Polaritons. Physical Review Letters 2013, 110, 236601.

39 Agnihotri, P.; Dhakras, P.; Lee, J. U.. Bipolar Junction Transistors in Two-Dimensional WSe 2 with Large Current and Photocurrent Gains. Nano Letters 2016, 16, 4355-4360.

40. Perez-Wurfl, I.; Krutsinger, R.; Torvik, J. T.; Van Zeghbroeck, B. .4H-SiC Bipolar Junction Transistor with High Current and Power Density. Solid-State Electronics ,2003,47 , 229-231.

41. Krishnaswami, S.; Agarwal, A.; Ryu, S.-H.; Capell ,C.; Richmond, J.; Palmour, J.; Balachandran, S.; Chow, T.P.; Bayne ,S.; Geil ,B.; Jones, K.; Scozzie ,C.. 1000-V, 30-A 4H-SiC BJTs With High Current Gain. IEEE ELECTRON DEVICE LETTERS, 2005,VOL. 26, NO. 3.

42. Crabbe, E.F.; Meyerson, B.S.; Stork, J.M.C. ; Harame, D.L., Vertical Profile Optimization of Very High Frequency Epitaxial Si- and SiGe-Base Bipolar Transistors. IEEE, 1993, IEDM 93,83-86.

43. Iyer, S.S.; Patton, G.L.; Stork, J.M.C.; Meyerson, B.S.; Harame, D.L.. Heterojunction Bipolar Transistors Using Si-Ge Alloys. IEEE TRANSACTIONS ON ELECTRON DEVICES, 1989,VOL. 36, NO. 10.

44. Xing, Huili; Chavarkar, P. M.; Keller, S.; DenBaars, S. P.; Mishra, U. K.. Very High Voltage Operation $(>330 \mathrm{~V})$ with High Current Gain of AlGaN/GaN HBTs. IEEE ELECTRON DEVICE LETTERS 2003,VOL. 24, NO. 3. 
(a)

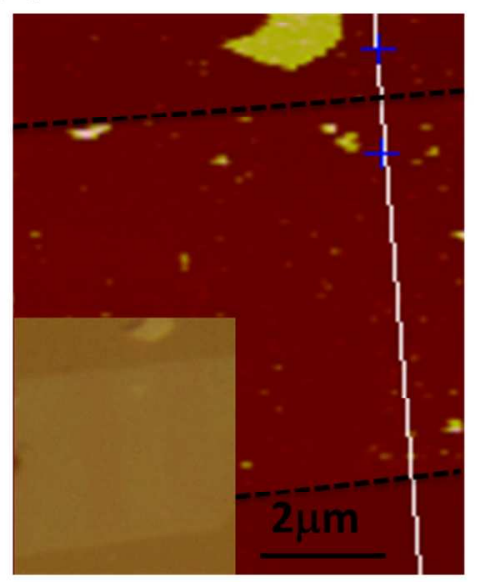

(c)

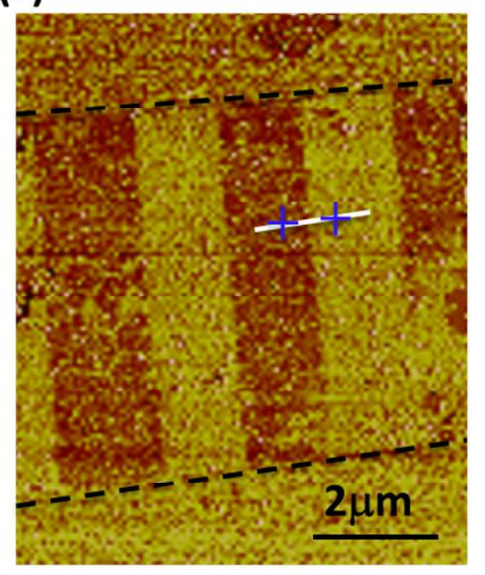

(b)

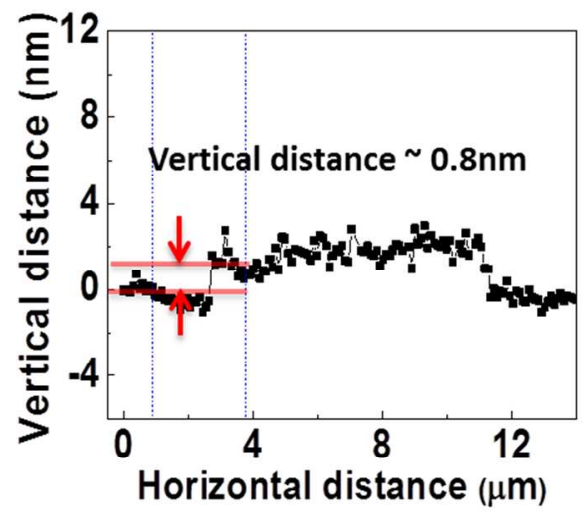

(d)

\section{Vertical distance deviation $1 \AA$}

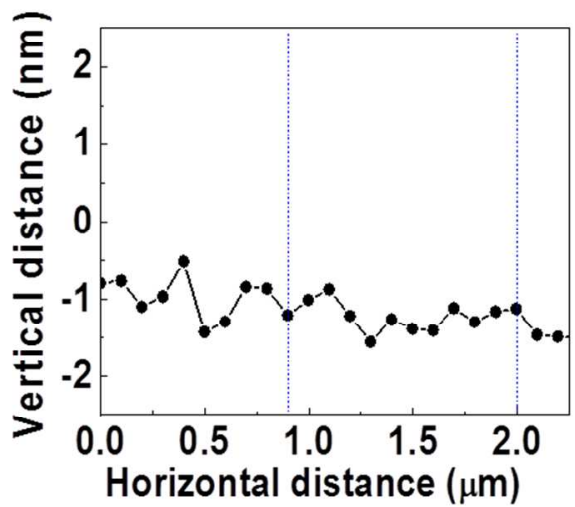

(e)

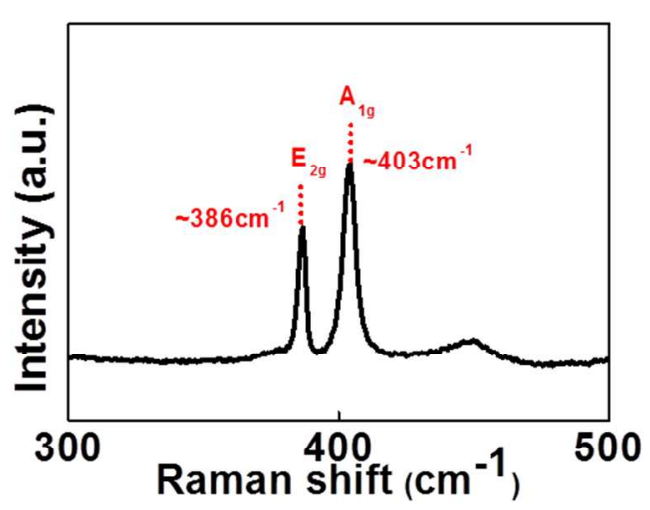

(f)

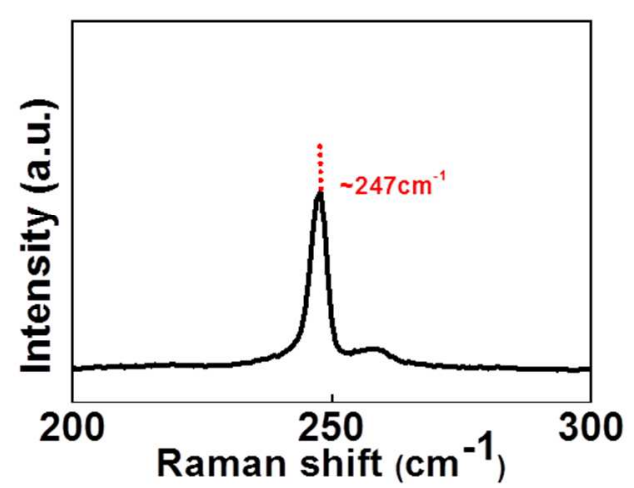

Figure 1. Characterization of the 2D lateral $\mathrm{WSe}_{2}-\mathrm{MoS}_{2}$ heterostructure. (a) $\mathrm{AFM}$ image of a lateral $\mathrm{WSe}_{2}-$ $\mathrm{MoS}_{2}$ heterostructure ribbon. Inset shows the corresponding optical micrograph. (b) The height profile of the 2D lateral heterostructure marked in (a), showing a thickness of about $0.8 \mathrm{~nm}$. (c) Phase image of AFM. The phase image shows clear contrast between $\mathrm{WSe}_{2}$ and $\mathrm{MoS}_{2}$. (d) The height profile of the lateral junction between two materials marked in (c), showing an ultra smooth interface. (e) Raman spectra for the $\mathrm{MoS}_{2}$ of the lateral WSe $2-\mathrm{MoS}_{2}$ heterostructure. (f) Raman spectra for the $\mathrm{WSe}_{2}$ of the lateral $\mathrm{WSe}_{2}-\mathrm{MoS}_{2}$ heterostructure. 
(a)

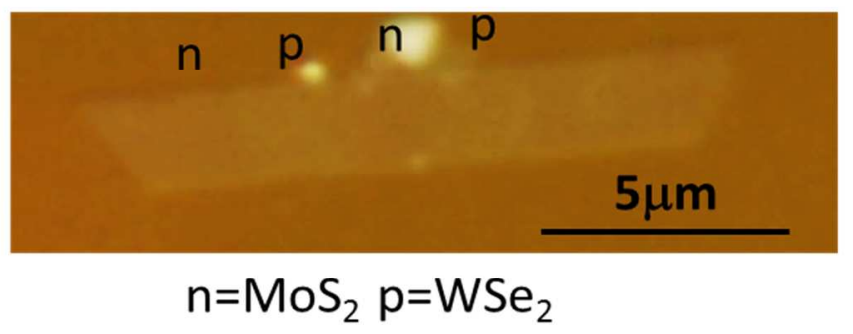

(c)

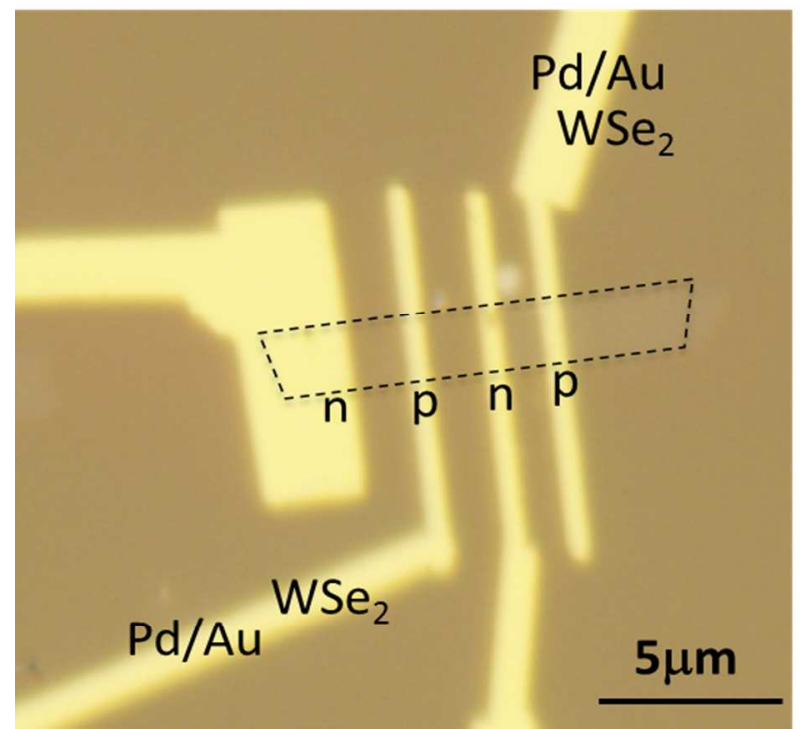

\section{(b)}

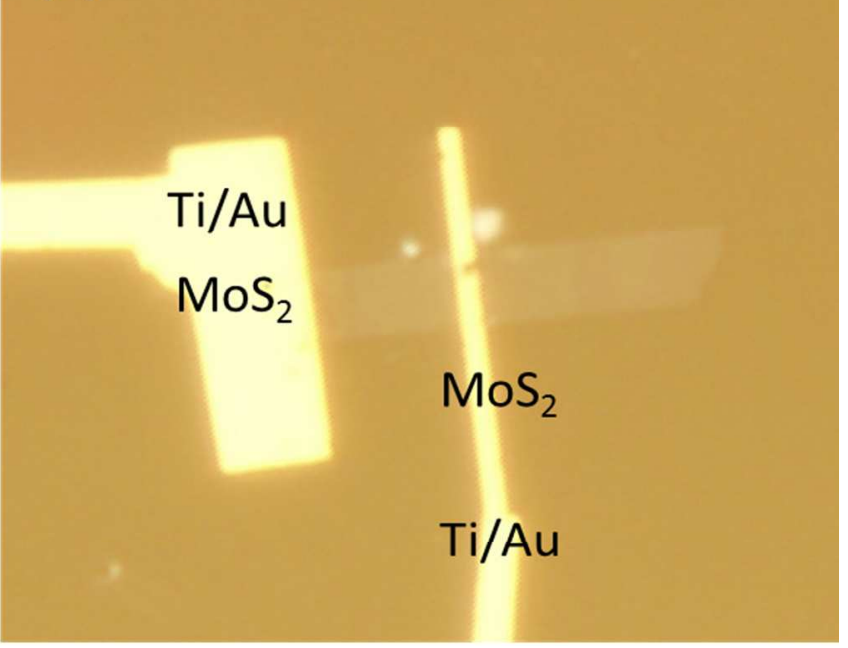

(d)

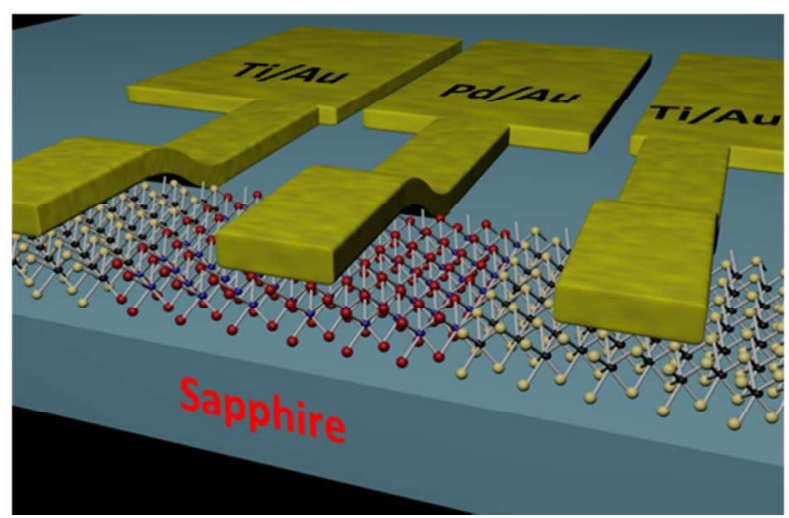

Figure 2. Device fabrication of two-dimensional lateral quasi-heterojunction bipolar transistors. (a) The optical micrograph of a lateral heterostructure ribbon where n-type material is $\mathrm{MoS}_{2}$ and p-type material is $\mathrm{WSe}$. (b) The optical micrograph of the metal $\mathrm{Ti} / \mathrm{Au}$ depositing on the top of $\mathrm{MoS}_{2}$ in the lateral heterostructure ribbon. (c) The optical micrograph of the lateral quasi-heterojunction bipolar transistor, showing an n-p-n-p lateral heterojunction. Black dashed box marks the position of the lateral hetero-structure ribbon. (d) Schematic plot of a $2 \mathrm{D}$ quasi-heterojunction bipolar transistor. The yellow ribbons are $\mathrm{MoS}_{2}$ monolayers and the red ribbon is $\mathrm{WSe}_{2}$ monolayer. Ti/Au metal layers are designed to deposit on $\mathrm{MoS}_{2}$ while $\mathrm{Pd} / \mathrm{Au}$ contacts with $\mathrm{WSe}_{2}$. 
(a)

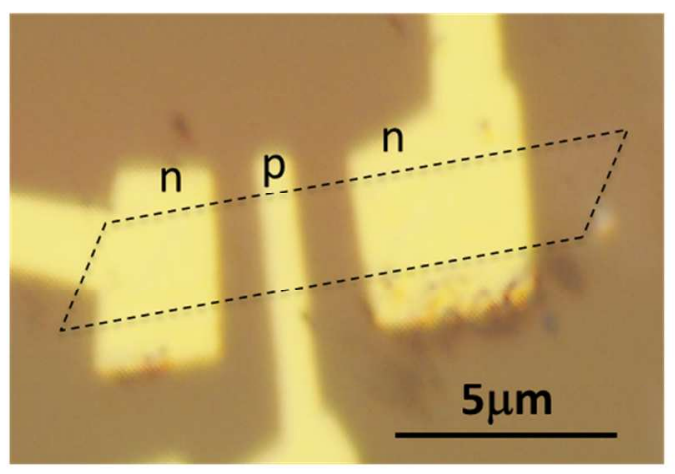

(c)

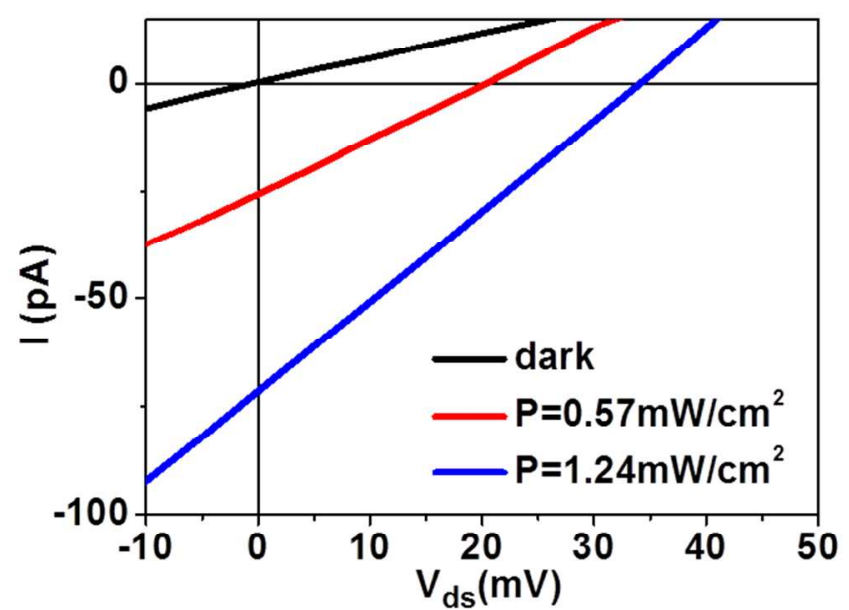

(b)

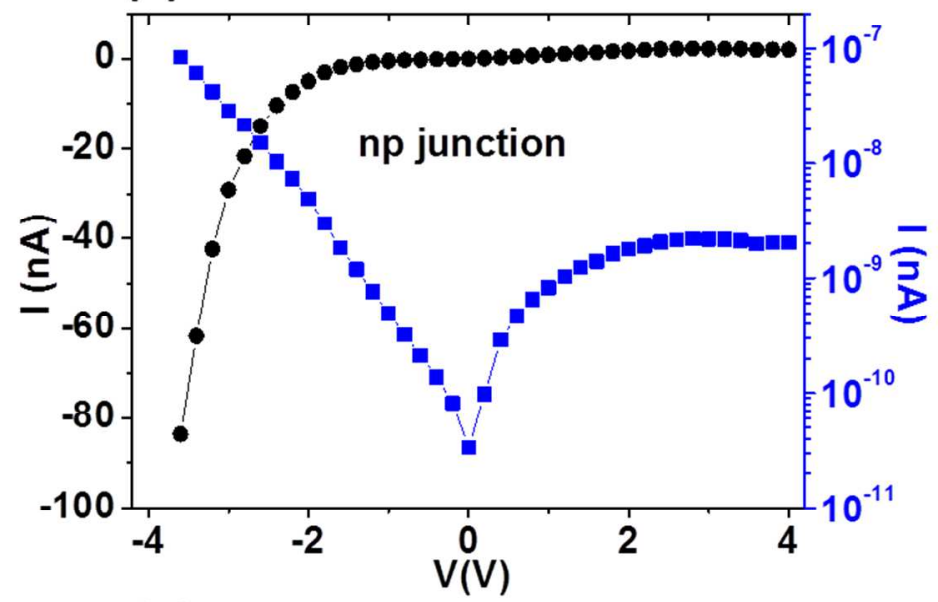

(d)

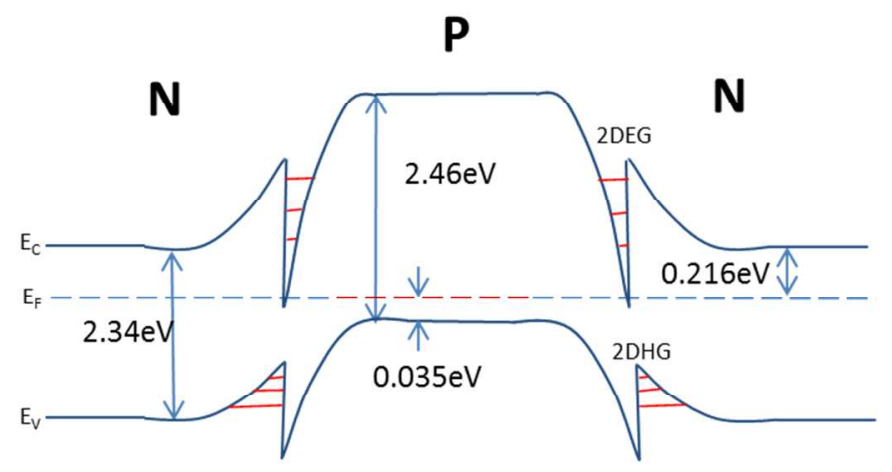

Figure 3. Electrical characterization of one of our n-p-n two-dimensional lateral structure. (a) The optical micrograph of a lateral n-p-n quasi-heterojunction bipolar transistor. (b) The I-V curve for the n-p junction. (c) The photo-response of the p-n junction in dark and under illumination at different intensities. (d) Band diagram of the n-p-n lateral quasi-heterojunction bipolar transistor. The electrical bandgap of the $\mathrm{MoS}_{2}$ monolayer is about $2.34 \mathrm{eV}$ while the bandgap of the $\mathrm{WSe}_{2}$ is around $2.46 \mathrm{eV}$. 
(a)

(b)
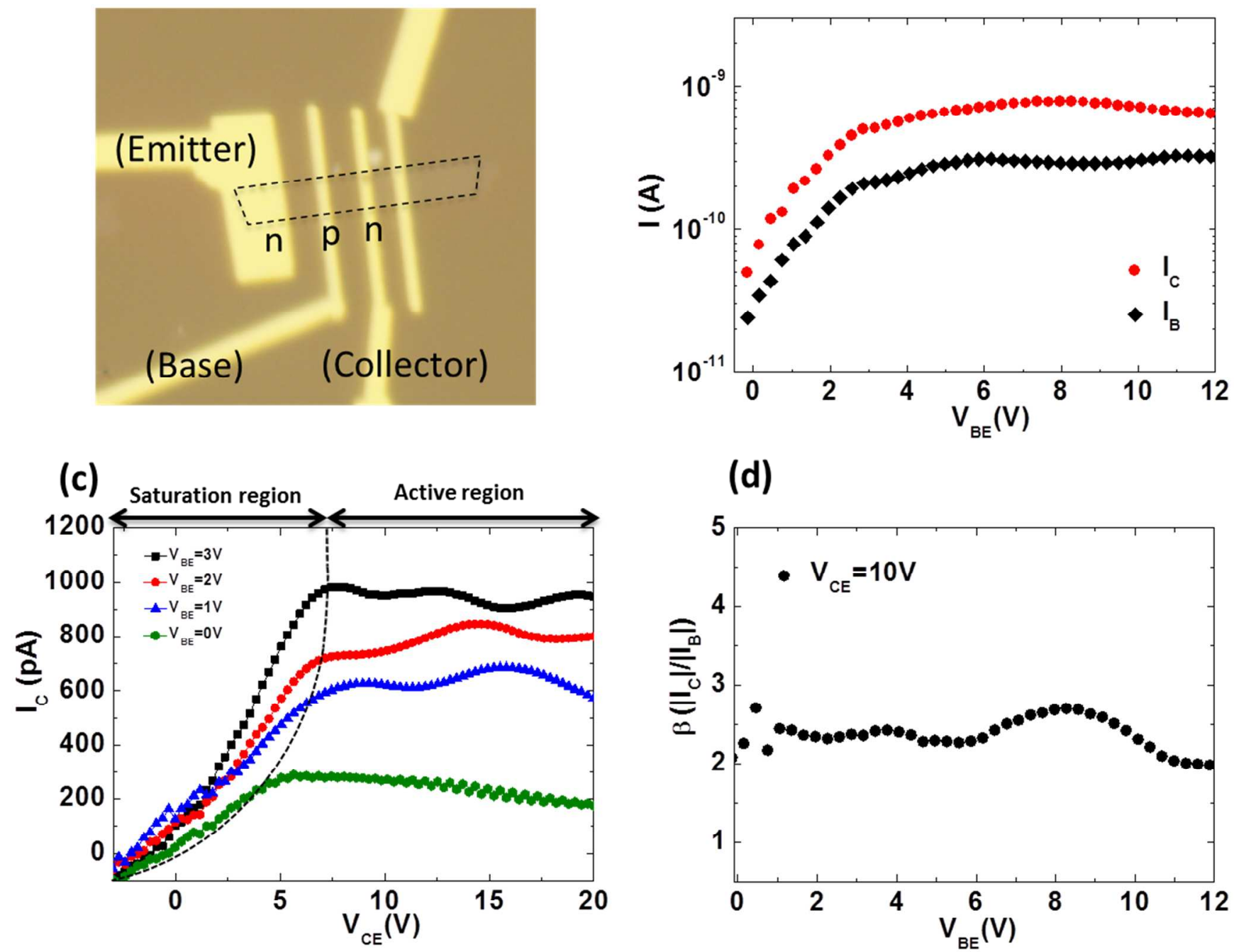

(d)

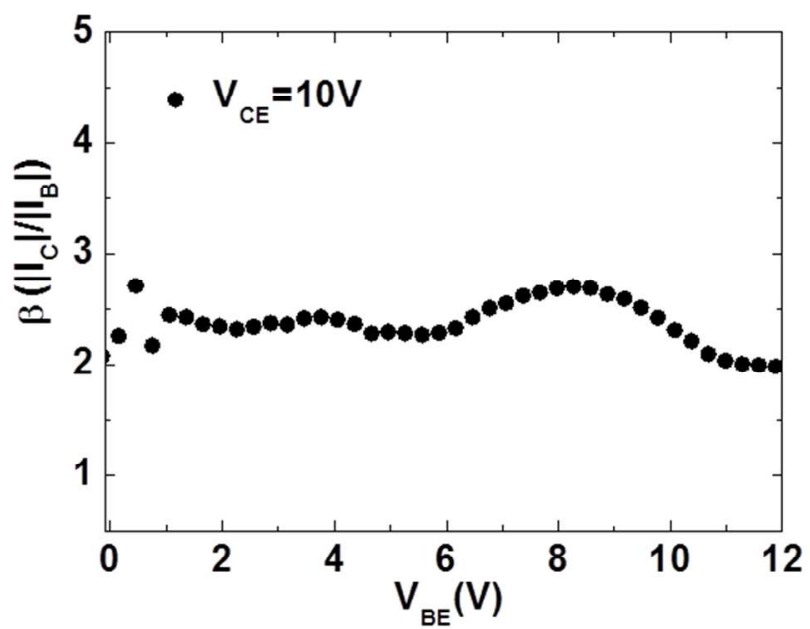

Figure 4. Electrical characterization of an n-p-n lateral quasi-heterojunction bipolar transistor. (a) The optical micrograph of the n-p-n lateral quasi-heterojunction bipolar transistor. (b) Gummel plot for the measured n-p-n quasi-heterojunction bipolar transistor biased in the common-emitter configuration. The collector and base currents are shown as a function of $\mathrm{V}_{\mathrm{BE}}$ at a fixed output voltage of $\mathrm{V}_{\mathrm{CE}}=+10 \mathrm{~V}$. (c) The output characteristics of the lateral $n-p-n$ quasi-heterojunction bipolar transistor at different $V_{B E}$ values. (d) Common-emitter current gain $(\beta)$ as a function of $\mathrm{V}_{\mathrm{BE}}$ at $\mathrm{V}_{\mathrm{CE}}=10 \mathrm{~V}$. 
(c)

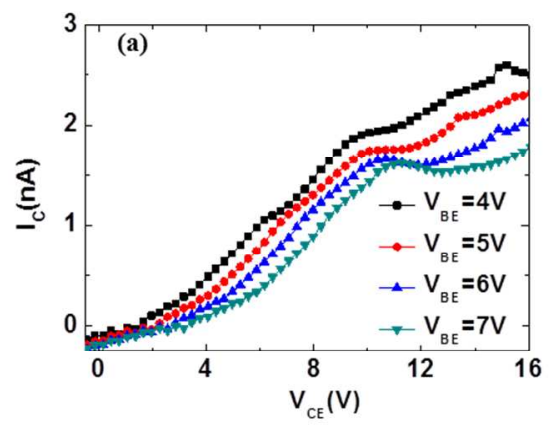

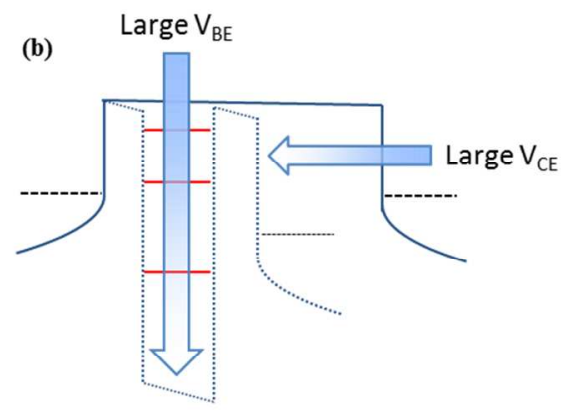

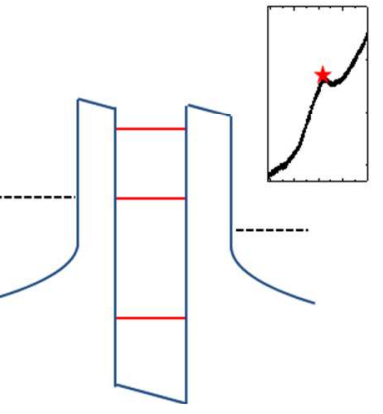

(d)

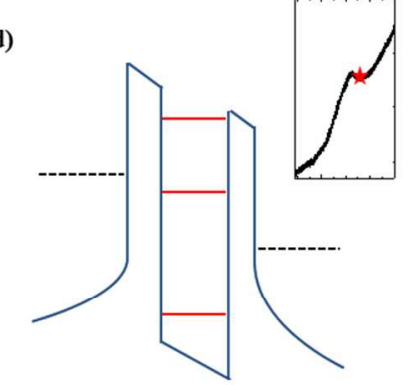

$\mathrm{MoS}_{2}$ Fermi level ......

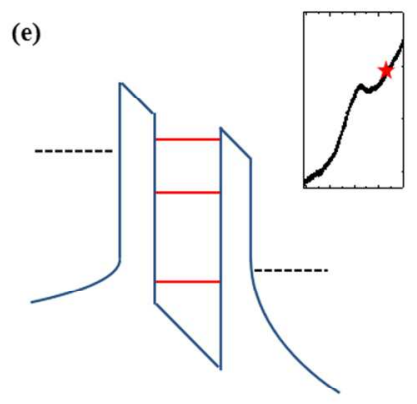

Figure 5. Electrical characterization of an n-p-n lateral quasi-heterojunction bipolar transistor in large base and collector bias and the corresponding schematic energy band diagram. (a) The output characteristics of the lateral n-p-n quasi-heterojunction bipolar transistor at relatively large $V_{\mathrm{BE}}$ values. (b) The formation of confined state by increasing base and collector bias voltages, illustrating the deeper well by the base voltages and the decrease of the base length by the collector voltages. (c), (d) and (e) Relation between energy confined states and source Fermi level in $\mathrm{MoS}_{2}$ and their corresponding output characteristics for the three cases of $\mathrm{V}_{\mathrm{CE}}$ voltages applied respectively. 
(a)

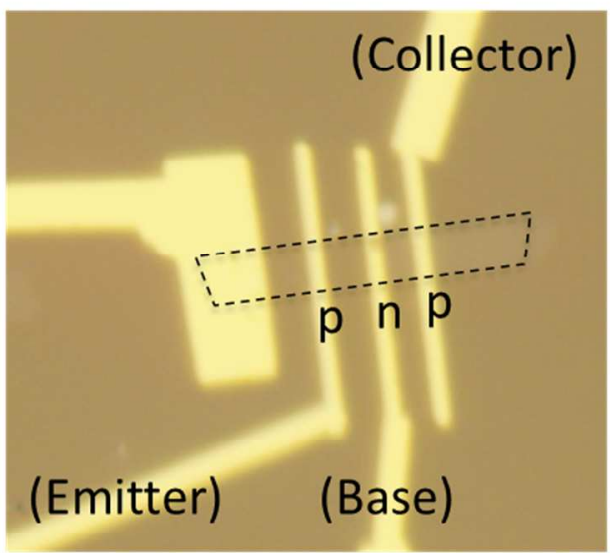

(c)

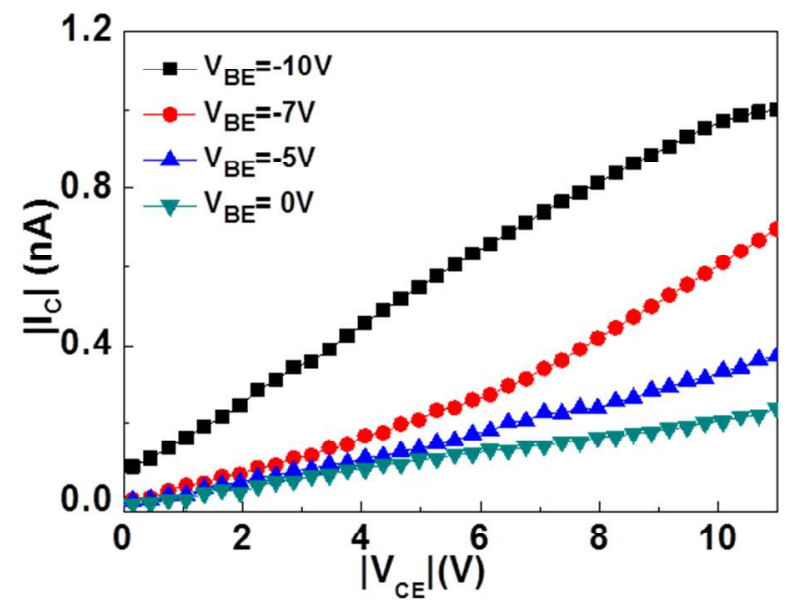

(b)

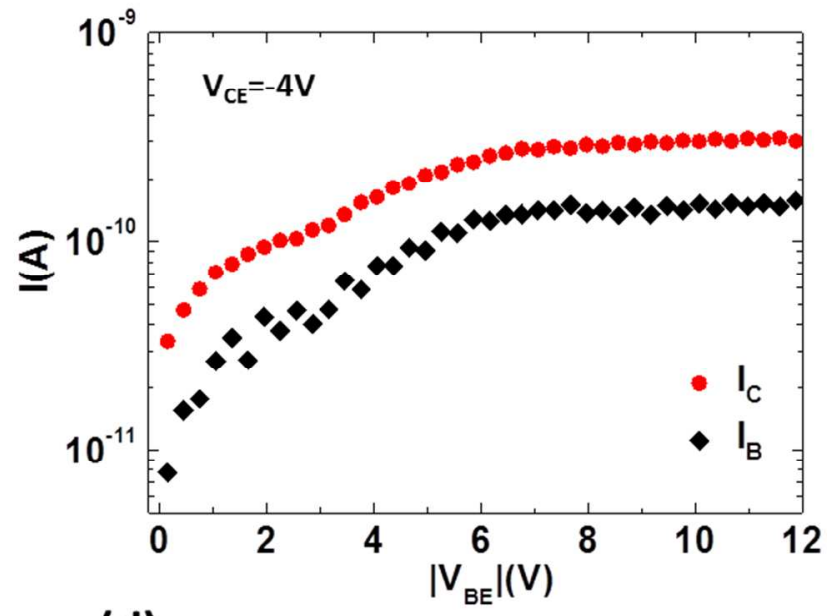

(d)

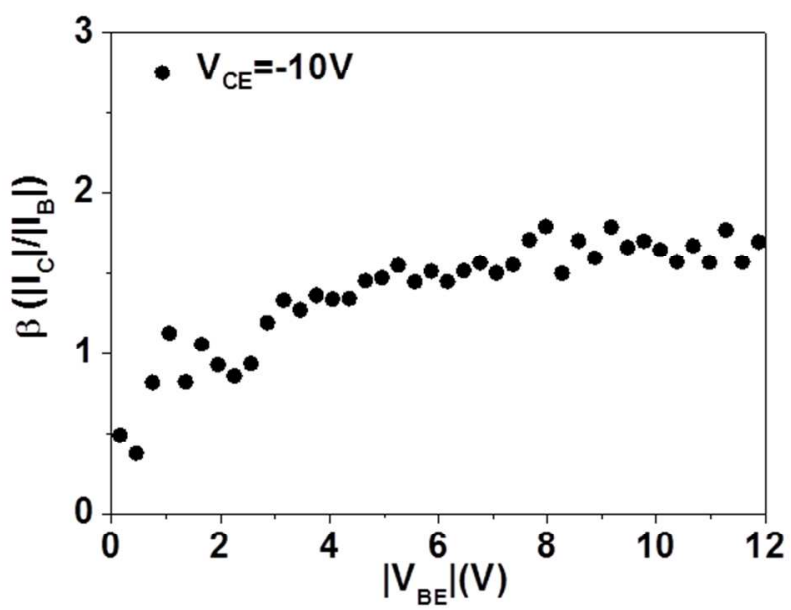

Figure 6. Electrical characterization of a p-n-p lateral quasi-heterojunction bipolar transistor. (a) The optical micrograph of the p-n-p lateral quasi-heterojunction bipolar transistor. (b) Gummel plot for the measured p-n-p quasi-heterojunction bipolar transistor biased in the common-emitter configuration. The collector and base currents are shown as a function of $\mathrm{V}_{\mathrm{BE}}$ at a fixed output voltage of $\mathrm{V}_{\mathrm{CE}}=-4 \mathrm{~V}$. (c) The output characteristics of the lateral p-n-p quasi-heterojunction bipolar transistor at different $V_{\mathrm{BE}}$ values. (d) Common-emitter current gain $(\beta)$ as a function of $\mathrm{V}_{\mathrm{BE}}$ at $\mathrm{V}_{\mathrm{CE}}=-10 \mathrm{~V}$. 
1

2

3

4

5

6

7

8

9

10

11

12

13

14

15

16

17

18

19

20

21

22

23

24

25

26

27

28

29

30

31

32

33

34

35

36

37

38

39

40

41

42

43

44

45

46

47

48

49

50

51

52

53

54

55

56

57

58

59

60

Table 1. The comparison of device performance and properties between this work and devices made from other materials.

\begin{tabular}{|c|c|c|c|c|c|c|}
\hline Ref. & Material & Type & $\begin{array}{c}\text { Current } \\
\text { gain }\end{array}$ & $\begin{array}{c}\text { Current } \\
\text { density }\left(\mathrm{A} / \mathrm{cm}^{2}\right) \\
\text { at room } \\
\text { temperature }\end{array}$ & Thickness & Structure \\
\hline$[40]$ & SiC BJT & NPN & 9 & 102.91 & $3250 \mathrm{~nm}$ & vertical \\
\hline$[41]$ & $\mathrm{SiC}$ BJT & $\mathrm{NPN}$ & 40 & 333 & $17.5 \mu \mathrm{m}$ & vertical \\
\hline$[42]$ & $\begin{array}{c}\text { Si BJT } \\
\text { SiGe HBT(Ge:0 15\%) } \\
\text { SiGe HBT(Ge:0 25\%) }\end{array}$ & $\mathrm{NPN}$ & $\begin{array}{c}70 \\
415 \\
440\end{array}$ & Not applicable & $\sim 300 \mathrm{~nm}$ & vertical \\
\hline$[43]$ & $\begin{array}{c}\text { Si BJT } \\
\text { Si BJT } \\
\text { SiGe } \mathrm{HBT}(\mathrm{Ge}: 10 \%)\end{array}$ & $\begin{array}{l}\text { NPN } \\
\text { PNP } \\
\text { PNP }\end{array}$ & $\begin{array}{c}16 \\
7 \\
45\end{array}$ & $\begin{array}{c}4.4 \\
3.3 \\
20.3\end{array}$ & $\begin{array}{l}\sim 400 \mathrm{~nm} \\
\sim 400 \mathrm{~nm} \\
\sim 450 \mathrm{~nm}\end{array}$ & vertical \\
\hline$[44]$ & $\mathrm{Al}_{0.05} \mathrm{GaN} / \mathrm{GaN}$ HBT & $\mathrm{NPN}$ & 18 & 1000 & $8.25 \mu \mathrm{m}$ & lateral \\
\hline $\begin{array}{l}\text { This } \\
\text { work }\end{array}$ & $\mathrm{MoS}_{2} / \mathrm{WSe}_{2} \mathrm{HBT}$ & $\begin{array}{l}\text { NPN } \\
\text { PNP }\end{array}$ & $\begin{array}{l}3 \\
2\end{array}$ & $\begin{array}{c}0.0126 \\
0.004\end{array}$ & $0.8 \mathrm{~nm}$ & lateral \\
\hline
\end{tabular}




\section{Table of Contents}
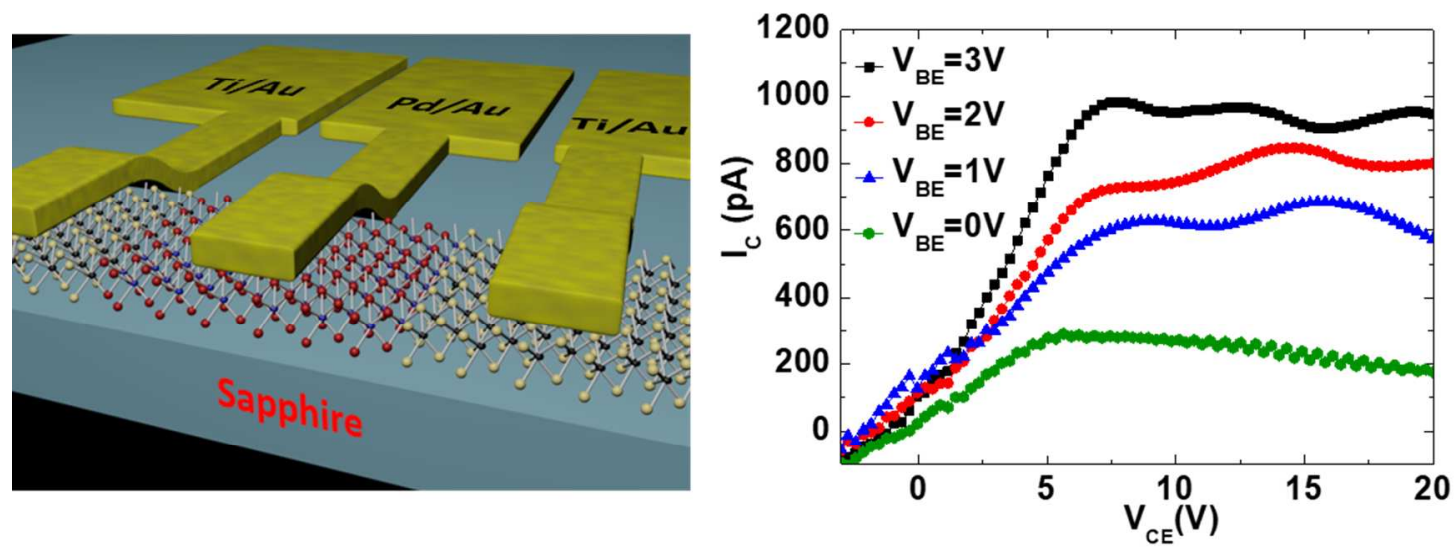